\title{
CONSTANTINO I Y LA SANTIDAD IMPERIAL: CONSTRUCCIÓN Y ROL POLÍTICO DE LA FIGURA DE “SAN CONSTANTINO”
}

\author{
Victoria Gerhold
}

(Consejo Nacional de Investigaciones Científicas y Técnicas. Argentina)

Resumen: La figura de Constantino I, el primer emperador santo de la tradición Ortodoxa, continuó siendo aún mucho después de su muerte objeto de reformulaciones y reinterpretaciones literarias -en especial historiográficas y hagiográficas- que intentaron adaptar su imagen a las diferentes circunstancias políticas que atravesó el Imperio Romano de Oriente. En el presente artículo se realiza una aproximación a algunas de las tradiciones bizantinas en torno a la santidad de Constantino I, con el objetivo de analizar en primera instancia cuáles fueron los elementos que orientaron la definición de su imagen de emperador santo, y, en segunda instancia, cuál fue el rol político que la santidad de Constantino I jugó en diferentes contextos históricos.

Palabras clave: Constantino I, San Constantino, santidad imperial, leyenda pagana, leyenda arriana.

\section{CONSTANTINE I AND IMPERIAL SANCTITY: CONSTRUCTION AND POLITICAL ROLE OF THE FIGURE OF "SAINT CONSTANTINE"}

Abstract: The figure of Constantine I, the first holy emperor of the Orthodox tradition, continued to be after his death the objet of reformulations and literary reinterpretations -mainly by historiography and hagiography- that attempted to adapt his image to the different political circumstances of the Eastern Roman Empire. This article develops some of the byzantine traditions concerning the holiness of Constantine I in order to establish, in the first place, which elements orientated the definition of his image of holy emperor and, in the second place, which was the political role that Constantine's holiness played in different political contexts.

Key words: Constantine I - Saint Constantine - imperial sanctity - pagan legend - Arian legend.

Recibido: 8.01.13 - Aceptado: 21.03.13 
Correspondencia: VICTORIA GeRHOLD. E-mail: victoria_gerhold@hotmail.com Licenciada en Historia. Universidad Nacional del Sur. Argentina.

Becaria doctoral del CONICET.

Alumna doctoral en co-tutela entre la Universidad de Buenos Aires y la Sorbona. París.

Directores de tesis: Pablo Ubierna (Buenos Aires) y Michel Kaplan (París)

\section{Introducción}

onstantino I es, sin duda, el mejor conocido de los emperadores santos de la tradición Ortodoxa. En tanto primer emperador cristiano, la definición de su santidad estaba investida de un profundo significado simbólico -tanto político como puramente religioso- para la Iglesia en particular y para la comunidad cristiana en general, y no es sorprendente por ende que Constantino haya sido uno de lo pocos emperadores santos en torno a los cuales se desarrolló un verdadero culto al interior de la Ortodoxia.

Sin embargo, la definición de su santidad no fue un proceso sencillo. Por el hecho mismo de haber jugado un rol tan significativo en la historia política del Imperio, Constantino había de convertirse necesariamente en una figura controvertida, tanto al interior de la polémica pagano-cristiana como al interior de las polémicas por la definición de la ortodoxia cristiana. Y, en gran medida, su definición como emperador santo estuvo fundada en esa dialéctica políticoreligiosa, que llevó a reformular de forma permanente las características que se juzgaba apropiado -y necesario- encontrar en la figura de san Constantino. La construcción de su santidad, en tal sentido, fue un proceso continuo y dinámico, que debió adaptarse a las diferentes épocas y a las diferentes exigencias políticas, y que debió responder a las varias tradiciones que pretendían poner en duda el modelo ideal del emperador santo.

De un modo general pueden identificarse tres grandes tradiciones con las cuales la definición cristiana y ortodoxa de san Constantino entabló una discusión en torno a su figura: la primera de ellas es la tradición pagana, responsable de la difusión de una suerte de leyenda negra del emperador a la que debió responder el cristianismo oriental; la segunda es la tradición arriana, la cual, a la manera del paganismo, difundió una versión difamatoria del emperador que había excluido su teología del marco de la ortodoxia; la tercera es la tradición papal, que, a 
diferencia de las anteriores, no fue considerada negativa en Oriente y por ende aceptada sin polémica alguna. Es en las dos primeras tradiciones -la pagana y la arriana- que nos detendremos en las páginas siguientes, a fin de considerar de qué manera influyeron en la definición de san Constantino que habría de cristalizar en la tradición Ortodoxa.

\section{Contra el paganismo}

Una de la dimensiones de la santidad de san Constantino era la de rey conversor, y se definía, en consecuencia, por oposición al paganismo. La santificación del primer emperador cristiano -en especial, del emperador que había dado el paso decisivo hacia la cristianización del Imperio- ${ }^{1}$ tenía como objeto insinuar la dimensión religiosa de toda su actividad política y exaltar su autoridad incluso por encima del título imperial. Para ello era necesario que la figura de Constantino y su período de reinado fuesen idealizados y fijados por la tradición cristiana como un modelo ejemplar de conducta: la definición de san Constantino que, en muchos aspectos, poco fundamento tuvo en el Constantino histórico, fue la tarea de varias generaciones de escritores cristianos.

La santificación de Constantino, como es sabido, debió mucho a la iniciativa de Eusebio, obispo de Cesárea, biógrafo oficial del emperador, cuya Vita Constantini puede ser considerada la única hagiografía imperial de época bizantina ${ }^{2}$. Eusebio fue responsable de establecer los temas principales de la leyenda cristiana de san Constantino -la revelación de la cruz antes de la batalla del Puente Milvio, el sueño en que Jesús esclareció el símbolo para el emperador-, que explicarían por revelación directa la conversión imperial ${ }^{3}$ y que se convertirían, al menos durante

${ }^{1}$ La leyenda de Filipo el Árabe afirmaba que había sido éste el primer emperador en profesar el cristianismo, aunque, a diferencia de Constantino, las creencias de Filipo el Árabe nunca se habrían hecho públicas. El rol político de esa leyenda, destinada a hacer coincidir la era cristiana con el inicio del Imperio, era así esencialmente distinto al de la tradición cristiana en torno a Constantino. Cf. G. Dagron, Empereur et prêtre. Étude sur le "césaropapisme" byzantin, Gallimard, Paris, 1996, p.143.

${ }^{2}$ La única con carácter oficial, ya que se conservan otras Vidas del emperador: $B H G 362 ; B H G$ 363; $B H G$ 365z, 366 y 366a; BHG 364; BHG 365; BHG 365n; además de otras versiones legendarias de la vida de Constantino. La hagiografía del emperador de Nicea Juan III Doukas Vatatzes, por otra parte, data de época post-bizantina; cf. G. Dagron, Empereur et prêtre, p.163.

${ }^{3}$ F. Winkelmann (ed.), Eusebius Werke, Band. 1.1: Über das Leben des Kaisers Konstantin [Die griechischen christlichen Schriftsteller], Akademie Verlag, Berlin, 1975, I.28-29. Para una síntesis actualizada de las discusiones en torno a la visión de Constantino, cf. R. Van Dam, Remembering Constantine at the Milvian Bridge, New York, Cambridge University Press, 2011, pp. 4-5. 
Victoria Gerhold: Constantino I y la santidad imperial: construcción y rol político ...

los primeros siglos del Imperio cristiano, en los puntos de referencia obligados de la tradición del primer emperador santo.

No es coincidencia, en tal sentido, que la mayor oposición al reconocimiento de Constantino como un emperador virtuoso y modelo de príncipes haya provenido de escritores paganos, quienes, concientes de la dimensión política de la santidad imperial, intentaron contrarrestar la leyenda cristiana mediante la difusión paralela de una "leyenda negra" de Constantino. Es por ello que durante los siglos tempranos del Imperio cristiano la imagen del emperador se vio definida y redefinida en función de la polémica pagano-cristiana: la santidad de Constantino sería sin duda uno de los ejes simbólicos más representativos de la transición religiosa del Imperio.

\subsection{La leyenda pagana}

La leyenda negra de Constantino I fue de origen temprano -se hallaba ya difundida durante la segunda mitad del siglo IV-, y se componía de una serie de temas que podían ser combinados de manera variable. Entre ellos es posible identificar al menos seis motivos recurrentes: a) el bajo origen familiar de Constantino ${ }^{4}$; b) la brutalidad y la codicia del emperador ${ }^{5}$; c) el asesinato del césar Crispo y de la emperatriz Fausta ${ }^{6}$; d) la conversión y el bautismo de

${ }^{4}$ Presente en fuentes paganas y en las refutaciones de autores cristianos. Cf. P. B. Kotter, Passio magni martyris Artemii, en Die Schriften des Johannes von Damaskos, vol. 5 [Patristiche Texte und Studien 29], De Gruyter, Berlin-New York, 1988, sección 41; C. de Boor (ed.), Georgii monachi chronicon, 2 vols., Teubner, Leipzig, 1904, p. 484; C. de Boor (ed.), Theophanis Chronographia, vol. 1, Teubner, Leipzig, 1883, p. 17; I. Bekker (ed.), Georgius Cedrenus opera [Corpus scriptorum historiae Byzantinae], Weber, Bonn, 1838-1839, vol. I, p. 476.

${ }^{5}$ F. Paschoud (ed.), Zosime. Histoire nouvelle, Les Belles Lettres, Paris, 1971-1989, II.29.1; Ch. Lacombrade (ed.), Simposion, en L'empereur Julien. Ouvres complètes, Les Belles Lettres, Paris, 1964, sección 36 ; Passio magni martyris Artemii, sección 43.

${ }^{6}$ F. Pichlmayr (ed.), Sexti Aurelii Victoris Liber de Caesaribus. Praecedunt Origo gentis Romanae et Liber de viris illustribus urbis Romae; subsequitur Epitome de Caesaribus, Leipzig, Teubner, 1911, 41.11-12 ; P. Courcelle y A. Loyen (eds.), Sidoine Apollinaire, Lettres, Les Belles Lettres, Paris, 1970, 5.8.2.; Zosime. Histoire nouvelle, II.29.2 ; J. Bidez y G. C. Hansen (eds.), Sozomenus. Kirchengeschichte [Die griechischen christlichen Schriftsteller 50], Akademie Verlag, Berlin, 1960, I.5.1-2; J. Bidez y L. Partmentier, The ecclesiastical history of Evagrius with the scholia. London, Methuen, 1898, p. 139; A. Adler (ed.), Suidae lexicon, Leipzig, Teubner, 1928-1935, entrada Kappa 2446; G.C. Hansen (ed.), Theodoros Anagnostes. Kirchengeschichte 2nd edn. [Die griechischen christlichen Schriftsteller der ersten Jahrhunderte. Neue Folge 3], Akademie Verlag, Berlin, 1995, I.2-4; T. Büttner-Wobst (ed.), Ioannis Zonarae epitomae historiarum libri xviii, vol.3 [Corpus scriptorum historiae Byzantinae], Weber, Bonn, 1897, pp. 12-13; I. Bekker (ed.), Michaelis Glycae annales [Corpus scriptorum historiae Byzantinae], Weber, Bonn, 1836, p.461; J.-P. Migne (ed.), Nicephorus Callistus Xanthopoulos, Historia ecclesiastica [Patrologiae cursus completus series Graeca], Paris, VII.35. 
Constantino $^{7}$; e) la fundación de Constantinopla ${ }^{8}$; f) la leyenda negra de santa Helena, a su vez integrada por una serie de subtemas propios?.

Algunos de esos temas tenían un interés marginal y accesorio -como es el caso de a), b), e) y f)-, y no se los encuentra en todas las versiones (o refutaciones) de la leyenda negra. Un tema como c), en cambio, constituía una referencia casi obligada, no por su interés individual, sino esencialmente porque era funcional al tema d), el más importante y el verdadero fundamento de la leyenda negra: la versión pagana de la conversión y el bautismo de Constantino I.

El Epitome de Caesaribus, escrito hacia fines del siglo IV, y la Historia Nova de Zósimo, escrita a principios del siglo VI pero probablemente basada en una obra de Eunapio de la segunda mitad del siglo IV, constituyen algunos de los testimonios directos más importantes de la tradición pagana en torno a Constantino I. El objetivo esencial era siempre el mismo: ofrecer una versión alternativa, y degradante, de la conversión del emperador, que contrarrestase la versión oficial de Eusebio de Cesárea y sus sucesores; el esquema narrativo, con pequeñas variaciones, era también el mismo: Constantino habría ordenado la muerte del césar Crispo, su hijo mayor, porque el joven había mantenido

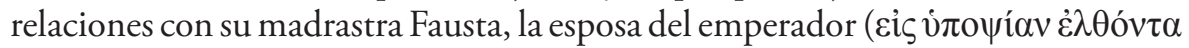

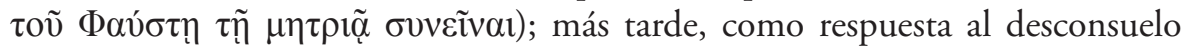
expresado por la emperatriz madre Helena por la muerte del mayor de sus nietos, Constantino habría decidido castigar también a Fausta con la muerte ${ }^{10}$;

7 Zosime. Histoire nouvelle, II.29.2-4; Sozomenus. Kirchengeschichte, I.5.1-2; The ecclesiastical history of Evagrius, p.139; Passio magni martyris Artemii, sección 43; Theodoros Anagnostes. Kirchengeschichte, I.2-4; Nicephorus Callistus Xanthopoulos, Historia ecclesiastica, VII.35.

${ }^{8}$ Zosime. Histoire nouvelle, II.30.1; The ecclesiastical history of Evagrius, p. 140.

${ }^{9}$ La leyenda negra de Santa Helena incluye al menos cuatro tremas: 1) su bajo origen familiar y profesión de cortesana (Passio magni martyris Artemii, sección 41); 2) el hecho de no haber estado casada con Constancio, el padre de Constantino (Passio magni martyris Artemii, sección 41 ); 3) su participación en los hechos que llevaron a la muerte de Fausta (Epitome de Caesaribus, 41.11-12; Zosime. Histoire nouvelle, II.29.2 ; Suidae lexicon, entrada Kappa 2446 ); 4) sus simpatías judías (Ioannis Zonarae epitomae historiarum, p.10; Michaelis Glycae annales, p. 462). El origen humilde de Santa Helena, y su unión extramatrimonial con Constancio I, no tienen, sin embargo, siempre carácter negativo. Cf., por ejemplo, The Origin of Constantine (Anonymus Valesianus pars prior [Origo Constantini], y la Guidi Vita Constantini (BHG 364), en S. N. C. Lieu y D. Montserrat (eds.), From Constantine to Julian: Pagan and Byzantine Views. A Source History, Londres - Nueva York, Routledge, 1996, pp. 43 y 108-109 respectivamente.

${ }^{10}$ Epitome de Caesaribus, 41.11-12; Zosime. Histoire nouvelle, II.29.2. El Epitome no menciona la relación entre Crispo y Fausta, encontrada raramente de forma explícita en las versiones paganas. Por el contrario, sugiere que Fausta calumnió a Crispo ante el emperador, sin explicar el motivo ni el carácter de la calumnia. 
sin embargo, arrepentido de sus crímenes, el emperador habría pedido luego a los sacerdotes paganos que lo purificasen, sólo para obtener de ellos una negativa: tales crímenes no tenían purificación posible ${ }^{11}$; por casualidad Constantino se habría encontrado poco después con un cristiano, Egipto de Iberia (Airv $\pi \tau$ tó $\tau ı \varsigma$ غ̇ $\left.\xi^{\prime} I \beta \eta \rho i ́ \alpha\right)^{12}$, quien le habría explicado que su religión perdonaba todos los pecados y absolvía de todo crimen a quien se convirtiese; habiendo creído en sus palabras, el emperador habría abandonado rápidamente las creencias tradicionales para recibir las que le ofrecía Egipto ${ }^{13}$.

En El Simposio, Juliano el Apóstata ratificaba de manera menos explícita la versión pagana de la conversión. Sin mencionar las muertes del césar y la emperatriz, Juliano insinuaba la importancia del perdón de los pecados ofrecida por el cristianismo para la conversión de Constantino:

"En lo que concierne a Constantino, no lograba encontrar entre los dioses el modelo de su propia vida; pero cuando hubo visto a Placer, que no estaba muy distante, corrió hacia ella. Ella lo recibió tiernamente y lo abrazó, y luego de embellecerlo y vestirlo con un traje multicolor lo condujo hasta Incontinencia. Allí también encontró a Jesús, que había establecido su morada junto a ella y proclamaba a todos cuantos se acercaban: “ ¡El que sea un seductor, el que sea un asesino, el que sea sacrílego e infame, que se acerque sin miedo! Con esta agua lo limpiaré y enseguida lo purificaré. E incluso si se hace culpable de los mismos pecados por segunda vez, que no haga más que golpear su pecho y su cabeza y lo purificaré por segunda vez. Y hacia él se acercó Constantino gustosamente (...)”14.

Otros temas completaban, eventualmente, la imagen de Constantino definida por la leyenda negra. "Ahora que el Imperio había caído en manos de Constantino, éste ya no ocultaba su malvado carácter y su inclinación al vicio, sino que actuaba como le placía, sin ningún control"15, según la explicación de Zósimo, para quien el reinado de Constantino había marcado el punto de inflexión en la decadencia del Imperio Romano. El motivo de "su inclinación al vicio” era enfatizado también por Juliano, quien prestaba al emperador algunas

\footnotetext{
${ }^{11}$ Zosime. Histoire nouvelle, II.29.3.

${ }^{12}$ Identificado con el obispo Osios de Córdoba, consejero de Constantino en el concilio de Nicea I.

${ }^{13}$ Zosime. Histoire nouvelle, II.29.4.

${ }^{14}$ Simposion, 38.

${ }^{15}$ Zosime. Histoire nouvelle, II.29.1.
} 
palabras de dudosa moralidad para responder, en su sátira de los césares, a la pregunta de Hermes sobre cuál había sido su ambición en vida: “Amasar una gran fortuna', respondió [Constantino], 'y luego gastarla con liberalidad para satisfacer mis propios deseos y los deseos de mis amigos"'16.

La alusión a los asesinatos de Crispo y Fausta, por su parte, no sólo resultaba útil para explicar la conversión de Constantino. Según la lógica del relato de Zósimo, los crímenes del emperador eran la causa indirecta de la fundación de Constantinopla: forzado por las circunstancias a convertirse a la fe cristiana, el emperador habría rehusado participar a partir de entonces en las antiguas ceremonias paganas, lo cual le habría granjeado el odio de los habitantes de Roma; en consecuencia, "incapaz de soportar la condena de casi todos los habitantes, buscó una ciudad que pudiese contraponerse a Roma, en la cual pudiera construirse un palacio" ${ }^{17}$. De esa manera Constantinopla, símbolo del nuevo orden cristiano, quedaba asociada a los crímenes del emperador y por ende estigmatizada desde su origen ${ }^{18}$.

\subsection{La leyenda cristiana}

Los autores cristianos no se detendrían a refutar, sin embargo, más que ocasionalmente los temas secundarios de la leyenda negra. El verdadero centro de la polémica estaría dado en realidad por el único tema que verdaderamente podía poner en duda la santidad del emperador y degradar así su autoridad política: las circunstancias de su conversión y su bautismo. Algunos de los sucesores inmediatos de Eusebio de Cesárea optaron por ignorar las provocaciones paganas y ratificar la versión tradicional de la Vita Constantini. Así, Sócrates retomaba el motivo de la visión de la cruz en el puente Milvio y el sueño en el que Jesús explicaba al emperador su significado simbólico, y ratificaba el bautismo en Nicomedia, también recordado por Teodoreto de Ciro ${ }^{19}$. Sozómeno evocaba igualmente la revelación en el Puente Milvio, el sueño profético y el bautismo en Nicomedia, pero se detenía además a refutar la versión pagana:

${ }^{16}$ Simposion, 36.

${ }^{17}$ Zosime. Histoire nouvelle, II.30.1

${ }^{18}$ Dagron G., Constantinople Imaginaire. Etudes sur le recueil des Patria, Presse Universitaire de France, Paris, 1984, pp.93-96.

${ }^{19}$ P. Maraval y P. Périchon, Socrate de Constantinople, Histoire ecclésiastique (Livres I-VII), Éditions du Cerf, Paris, 2004-2007, I.2 y I.39; L. Parmentier y F. Scheidweiler, Theodoret. Kirchengeschichte [Die griechischen christlichen Schrifsteller 44], Akademie Verlag, Berlin, 1954, p. 89. 
"No ignoro que los helenos dicen que Constantino, tras haber ordenado la muerte de algunos parientes cercanos, y especialmente

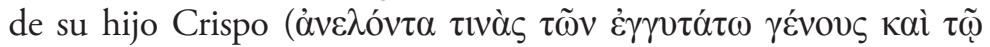

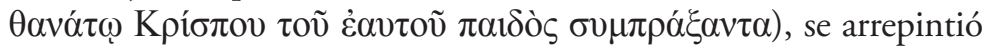
de sus perversas acciones y consultó al filósofo Sopater, jefe de la escuela de Plotino, sobre la forma de purificarse; éste le habría declarado sin embargo que no había purificación posible para tales crímenes. Hallándose muy turbado por esta respuesta, el emperador

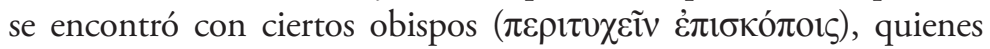
le dijeron que mediante el arrepentimiento y el bautismo sería purificado de todos sus crímenes; [el emperador] se mostró encantado con sus palabras y admiró su doctrina y se convirtió en cristiano y guió a sus súbitos hacia la fe. Según me parece, esta historia es una invención de personas que quieren desacreditar a la fe cristiana" ${ }^{20}$.

Evagrio Escolástico, que no se ocupaba en su obra de la vida de Constantino y cuyas referencias al emperador eran circunstanciales, se detenía sin embargo a refutar la misma versión pagana de la conversión imperial:

"Zósimo, un seguidor de la abominable y corrupta religión de los helenos, en su resentimiento contra Constantino por haber sido el primer emperador que adoptó el cristianismo, abandonando la detestable superstición de los helenos, dice (...) que él miserablemente destruyó a su hijo Crispo y a su esposa

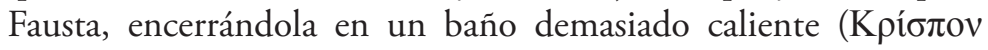

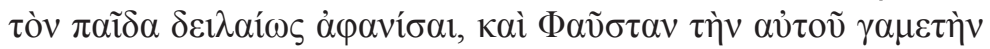

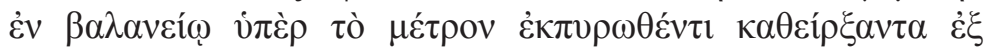
$\grave{\alpha} v \theta \rho \omega ́ \pi \omega \nu \mu \varepsilon \tau \alpha \gamma \alpha \gamma \varepsilon \tilde{\imath} v)$, y que luego de buscar purificación entre sus sacerdotes sin obtenerla se encontró con un egipcio llegado de

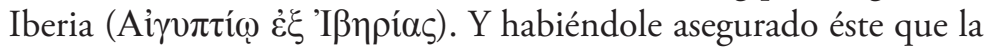
fe cristiana era capaz de purificar de todo pecado, compartió lo que el egipcio le ofrecía; abandonado a partir de entonces sus creencias ancestrales, se inició en la impiedad, como dice Zósimo. Y yo voy a mostrar cómo esto es en realidad falso (...)”.

"En prueba de que Constantino no destruyó ni a Fausta ni a Crispo (...) escucha la historia de Eusebio Pamphilio, que fue

${ }^{20}$ Sozomenus. Kirchengeschichte, I. V1-2. 
contemporáneo de Constantino y Crispo, y tuvo relación con ellos (...). Y al final de su historia [Eusebio] se expresa en los siguientes términos: 'el gran, victorioso Constantino, adornado con toda virtud piadosa, junto con su hijo Crispo, un príncipe muy amado de Dios y similar a su padre en todo sentido, obtuvo la legítima posesión del Este'. Eusebio, que sobrevivió a Constantino, nunca hubiera elogiado a Crispo en esos términos si éste hubiera sido destruido por su padre"21.

Teodoro Anagnostes, a la manera de Sozómeno, recordaba la revelación en el puente Milvio y el sueño del emperador, pero se veía igualmente en la necesidad de aclarar la falsedad de la versión pagana de la conversión:

"Algunos de entre los helenos difaman a Constantino, diciendo que asesinó a su hijo Crispo y a otros de sus parientes

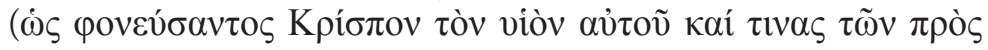
$\gamma \varepsilon \dot{v}$ ovs), y que sintiéndose después culpable y queriendo ser purificado de la mancha del sacrilegio pidió consejo al filósofo Sopatro, jefe de la herejía de Plotino. Habiéndole respondido éste que era imposible purificarlo de su crimen, Constantino se reunió

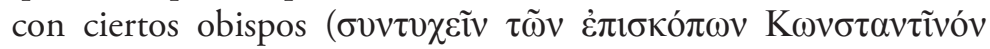
$\tau \iota \sigma \downarrow)$, quienes le dijeron que mediante el arrepentimiento y el bautismo lograría la purificación. Y así explican cómo adoptó la fe cristiana; pero, como ya fue dicho, mienten"22.

El eje de la polémica, por supuesto, era falso, porque las muertes de Crispo y Fausta no habían estado históricamente relacionadas a la conversión del emperador. Sin embargo, la damnatio memoriae que había pesado por decisión imperial sobre las circunstancias de la desaparición del césar y la emperatriz, y por ende la inexistencia de una versión oficial que explicase sus muertes ${ }^{23}$, había

21 The ecclesiastical history of Evagrius, p.139, retomado por Nicéphoro Kallistos Xanthopoulos, Historia ecclesiastica, XVI.42. El argumento, desde ya, no tiene en cuenta que la Historia Ecclesiastica de Evagrio fue completada antes del 325, cuando el césar Crispo aún vivía y estaba en buenos términos con Constantino.

${ }_{22}$ Theodoros Anagnostes. Kirchengeschichte, 1.2-4.

${ }^{23}$ Eusebio no menciona los hechos en la Vita Constantini, sin duda respetando la damnatio memoriae impuesta por el poder imperial. El análisis de las fuentes existentes, por otra parte, no ha permitido llegar a ninguna conclusión definitiva. Cf., por ejemplo, P. Guthrie, "The Execution of Crispus", Phoenix, 20 (1966), pp. 325-31; J. Rougé, "Fausta, femme de Constantin: criminelle ou victime?", Cahiers d'Histoire, 25 (1980), pp. 3-17; N. J. E. Austin, "Constantine 
dejado un vacío que era fácil llenar a través de una explicación ficticia y, llegado el caso, funcional a un interés político.

Ante la difusión de la leyenda negra, los autores cristianos se veían obligados a aceptar la lógica de los hechos planteada por el paganismo y a ensayar una refutación en los mismos términos ${ }^{24}$. Las referencias a la revelación del puente Milvio y al sueño profético ya no bastaban para explicar las circunstancias de la conversión de Constantino: era preciso demostrar además que las muertes de Crispo y Fausta estaban desvinculadas del hecho, y por ende que la conversión no había sido un acto de conveniencia desprovisto de fe. Es por eso que Sozómeno se detenía a enfatizar que "Crispo, por causa de quién, se dice, Constantino requirió ser purificado, no murió hasta el año número veinte del reinado de su padre; ocupó el segundo lugar en el Imperio y llevó el título de césar, y muchas leyes, promulgadas con su sanción a favor del cristianismo, todavía existen hoy" 25 ; por eso mismo Evagrio recordaba que "Teodoreto, en su historia, dice que Constantino participó del bautismo salvador en Nicomedia cerca del final de su vida, y que había pospuesto el rito todo ese tiempo por causa del deseo de que fuese realizado en el río Jordán"26; por eso Teodoro Anagnostes enfatizaba, luego de haber objetado la versión pagana de los hechos, que había sido "la señal de la cruz que fue vista en el cielo durante la campaña contra Majencio la que llevó [a Constantino] a convertirse al cristianismo" ${ }^{27}$. Si se lograbas preservar así las circunstancias ideales de la conversión del emperador, la leyenda cristiana de Constantino preserva su esencia ${ }^{28}$.

and Crispus", Acta Classica 23 (1980), pp. 133-8; T. Barnes, Constantine and Eusebius, Cambridge Mass., Harvard University Press, 1981, p. 220; H. A. Pohlsander, "Crispus: Brillant Career and Tragic End", Historia 33 (1984), pp. 79-106; J. W. Drijvers, "Flavia Maxima Fausta: Some Remarks", Historia, 41 (1992), pp. 500-6; D. Woods, "On the death of the Empress Fausta", Greece and Rome, 45 (abril 1998), pp. 70-86.

${ }^{24}$ Es interesante notar que las refutaciones cristianas no incluían nunca versiones alternativas para explicar las muertes de Crispo y Fausta. Ello se debía, en parte, al hecho de que los acontecimientos habían sido ocultados por la damnatio memoriae ordenada por el poder imperial, y en parte también al hecho de que al menos algunos autores cristianos habían llegado a admitir el relato de los acontecimientos difundido por el paganismo, aunque no estuviesen dispuestos a admitirlo públicamente. Cf. Sidoine Apollinaire, Lettres, 5.8.2.

${ }_{25}$ Sozomenus. Kirchengeschichte, I.V.2.

${ }^{26}$ The ecclesiastical history, p.141.

${ }^{27}$ Kirchengeschichte, 1.4 .

${ }^{28}$ A partir del siglo VII, con la incorporación de temas tomados de la Vita Silvestri, la conversión y el bautismo de Constantino iban a quedar definitivamente desvinculados de las muertes de Crispo y Fausta, y este tema iba a dejar de ser considerado peligroso para la imagen de Constantino. Sin embargo, el motivo iba a permanecer en las fuentes, a veces presentado con carácter positivo (se 
La figura de Constantino I -y en particular ciertos aspectos de su vida, como la delicada cuestión de su conversión y su bautismo- fueron así, en primer término, un motivo de enfrentamiento político entre paganos y cristianos. Desde ya, ninguna de las versiones del la vida del emperador se ajustaba exactamente a los hechos históricos, ninguna carecía de un alto grado de ficción retórica adecuada a sus necesidades y sus intereses, y es así que el enfrentamiento era mucho menos por la historia que por la leyenda constantineana. Esa polémica, propia de los primeros siglos del Imperio cristiano, habría de extinguirse sin embargo a medida que el paganismo decaía como fuerza política. A partir del siglo VI, el interés de los escritores y polemistas cristianos tenía otra orientación, y es en función de ella que la leyenda de Constantino habría de verse nuevamente reformulada para adaptarse a las circunstancias.

\section{Contra la herejía}

Otra de las dimensiones de la santidad de San Constantino era la de monarca ortodoxo y se definía, en esencia, por oposición al arrianismo. En tanto primer monarca cristiano, Constantino había sido el primer emperador en convocar y presidir un concilio ecuménico, y el primero en intervenir directamente en cuestiones teológicas. Las decisiones tomadas por el emperador en el concilio de Nicea I habían sido decisivas para el triunfo de una línea teológica -la de la consubstancialidad del Hijo- que habría de pasar a ser la ortodoxia imperial ${ }^{29}$, y era en función de esa ortodoxia que resultaba necesario reivindicar la santidad del emperador que la había consagrado.

Ese aspecto de la leyenda cristiana de Constantino era, sin embargo, particularmente delicado, no sólo por las falsas acusaciones que los arrianos -

admitían los crímenes y se afirmaba que Constantino tuvo razón en cometerlos, cf. Passio magni martyris Artemii, sección 45), y a veces mencionado sin juicio de valor alguno (no se lo atribuía a los paganos ni se intentaba refutarlo, cf. Suidae lexicon, entrada Kappa 2446; Ioannis Zonarae epitomae historiarum, vol. 3 p. 12; Michaelis Glycae annales, p.461). En paralelo se iba a desarrollar la versión cristiana del arrepentimiento de Constantino: el emperador habría cometido los crímenes (al menos el crimen contra Crispo) y se habría arrepentido, sólo que a diferencia del motivo pagano (en el que el arrepentimiento llevaba a la conversión) el arrepentimiento en la versión cristiana sólo llevaba a la dedicación de una estatua a la memoria de Crispo, erigida en Constantinopla. Cf. A. Cameron y J. Herrin (eds.), Constantinople in the early eighth century: the Parastaseis Syntomoi Chronikai, Brill, Leiden, 1984, sección 7; T. Preger, Scriptores originum Constantinopolitanarum, Teubner, Leipzig, 1907, II.93; G. Dagron, Constantinople Imaginaire, pp.93-96.

${ }^{29}$ Recién a partir de su ratificación en el Concilio de Constantinopla I (381), ya que algunos de los sucesores de Constantino I -Constancio (337-361) y Valente (364-378)- habían promovido el arrianismo. 
resentidos por su derrota en Nicea- habrían difundido acerca del emperador, sino porque Constantino no había mantenido tras el concilio una conducta estrictamente nicena. Los últimos ańos de su vida, en efecto, estuvieron marcados por una tolerancia notoria e incluso cierta inclinación hacia el arrianismo, probablemente como resultado de las influencias de los obispos orientales que lo aconsejaban, Eusebio de Cesárea y Eusebio de Nicomedia. En todo caso, la leyenda cristiana de Constantino tenía, en este caso, el desafío doble de proteger la imagen imperial de las tradiciones arrianas y de reformular algunos de los escasos hechos históricos que se conservaban sobre la vida del emperador.

\subsection{La leyenda arriana}

La tradición arriana en torno a Constantino I es difícil de reconstruir, porque sólo contamos con evidencias indirectas de su contenido: los fragmentos de la Historia Eclesiástica de Philostorgius, citados o comentados por otras fuentes ${ }^{30}$, y las varias refutaciones ortodoxas a supuestas afirmaciones sostenidas por los arrianos. A partir de esos escasos testimonios es posible identificar al menos dos temas que con cierta probabilidad eran propios de la definición arriana de Constantino.

El primero de ellos es el del bajo origen familiar del emperador ${ }^{31}$. La Artemii Passio, una de las fuentes que se supone hizo uso extensivo de la obra de Philostorgius, ponía en boca del Juliano el Apóstata algunas expresiones en ese sentido - “ [Y sabes] que nuestra familia es la más adecuada para reinar. En efecto, mi padre Constancio fue engendrado por mi abuelo Constancio con Teodora, la hija de Maximiano; Constantino, por otra parte, fue engendrado por él con Helena, una mujer del común, no mejor que una prostituta" ${ }^{32}$.

Es difícil, sin embargo, determinar en qué contexto de la obra de Philostorgius eran utilizadas estas palabras; el hecho de que fuesen atribuidas a Juliano el Apóstata, tal como reproduce probablemente con fidelidad la Artemii Passio, sugeriría que el autor no habría declarado compartirlas. En todo caso, existen testimonios cristianos que reafirman la difusión arriana de ciertos rumores en torno al bajo origen de Constantino, como son los de Teophanes y Cedrenus _"Otros de los arrianos (...) calumnian al gran Constantino diciendo que era de

\footnotetext{
${ }^{30}$ P. Amidon (trad.), Philostorgius: Church History, Atlanta, Society of Biblical Literature, 2007.

${ }^{31}$ El bajo origen del emperador era también un tema pagano, como se ha señalado ya. Autores como Teophanes y Cedrenus lo atribuyen tanto a los paganos como a los arrianos (Theophanis Chronographia, p. 17; Georgius Cedrenus opera, vol. I, p. 476).

${ }^{32}$ Passio magni martyris Artemii, sección 41.
} 
baja cuna: mienten también éstos. Su genealogía, por el contrario, era real desde antes de Diocleciano"33. También estas referencias presentan algunas dificultades. En primer lugar, la versión ofrecida por Cedrenus sigue tan de cerca de Teophanes que no es posible suponer que haya contado con una fuente independiente, con lo cual su testimonio no aporta datos nuevos. Teophanes, por otra parte, puede haber aludido a los arrianos en sentido genérico como enemigos de Constantino, quizás sin tener certeza de las palabras que les atribuía. El primero de los temas es así dudoso hasta cierto punto.

El segundo tema propio de la tradición arriana en torno a Constantino es el de los asesinatos de Crispo y Fausta. En este caso contamos con evidencias mucho más seguras: la Artemii Passio ofrece, en primer lugar, un relato de las muertes del césar y la emperatriz probablemente tomado de la obra de Philostorgius; extractos de la obra de Photios, del siglo IX, conservan por su parte amplias referencias a la obra de Philostorgius, entre las cuales se recoge la versión ofrecida para las muertes de Crispo y Fausta; la Opitz Vita Constantini, una versión de la vida del emperador de entre los siglos IX-XI, reproduce a su vez una versión de las muertes del césar y la emperatriz que atribuye directamente a Philostorgius; por último, la crónica de Zonaras del siglo XI ofrece, sin mencionar a su fuente, una versión que coincide con las anteriores y que fue probablemente tomada de Philostorgius, como lo reafirman otros datos de su obra que coinciden con información presente en la Historia Eclesiástica arriana.

Los cuatro testimonios difieren escasamente entre sí, y difieren a su vez relativamente poco de las versiones paganas: Fausta se habría enamorado del césar Crispo y habría requerido sus favores; el césar se los habría negado, y para vengarse la emperatriz habría convencido a Constantino de que el joven había intentado forzarla; el emperador habría castigado a Crispo con la muerte; tras haber descubierto por fin la falta de la emperatriz, habría decidido castigarla también con la muerte ${ }^{34}$.

Como se observa, la versión arriana presenta una sola particularidad: el motivo que se ofrece para explicar las muertes del césar Crispo y de la emperatriz Fausta.

${ }_{33}$ Theophanis Chronographia, p. 18; Georgius Cedrenus opera, vol. I, p. 476.

34 Passio magni martyris Artemii, sección 45; H.-G. Opitz, "Die Vita Constantini des Codex Angelicus 22,” Byzantion 9 (1934), pp 535-93, secciones 35-36; F. Winkelmann (post. J. Bidez) (ed.), Philostorgius. Kirchengeschichte (fragmenta ap. Photium) [Die griechischen christlichen Schriftsteller], Akademie Verlag, 1981, II.4; Ioannis Zonarae epitomae historiarum, pp. 12-13. 
Victoria Gerhold: Constantino I y la santidad imperial: construcción y rol político ...

De entre los escritos paganos, sólo la Historia Nova de Zósimo daba una razón explícita para sus muertes -la relación extramatrimonial mantenida por Crispo y su madrastra-, razón retomada por una fuente cristiana, la enciclopedia Suda del siglo X. La mayor parte de los escritores cristianos, sin embargo, no se detenía en los motivos de las muertes: Sozómeno, Evagrio, Teodoro Anagnostes, Miguel Glykas, se contentaban con mencionar los crímenes atribuidos a Constantino sin explicar la razón de la conducta del emperador, lo que sugiere que las fuentes paganas de las cuales tomaban la descripción de los hechos no habrían dado excesiva importancia a las motivaciones de Constantino. Bastaba, sin duda, con señalar las muertes para poder pasar rápidamente a la consecuencia que les atribuía el paganismo: la conversión del emperador.

Por el contrario, las versiones que retomaban a Philostorgius -la Artemii Passio, la Opitz Vita, y la crónica de Juan Zonaras- se detenían detalladamente sobre los motivos que Constantino habría tenido para cometer el asesinato de su esposa e hijo. Todas ellas ofrecían un relato inspirado, como es evidente, en el antiguo mito de Fedra: el amor de la emperatriz por el joven césar, el rechazo de éste, la difamación del joven ante Constantino por parte de Fausta, la muerte de Crispo por decisión del emperador, el descubrimiento de la verdad y el castigo de Fausta con la muerte. En ese marco resulta interesante considerar si la introducción del mito de Fedra -citado, por otra parte, de forma explícita por la Artemii Passio, la Opitz Vita y la crónica de Zonaras- se debía a una iniciativa exclusivamente arriana, o si alguna versión pagana hacía alusión explícita al mismo motivo. No parece posible, sin embargo, ofrecer una respuesta conclusiva al respecto. La forma en que Zósimo presenta los hechos es ambigua, pero no parece coincidir con el mito: por una parte, hace referencia clara a la existencia efectiva de una "relación"

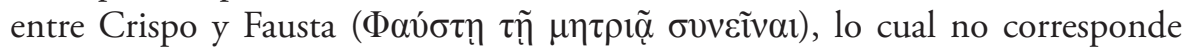
al desarrollo del mito; por otra parte, Zósimo no menciona el motivo mítico tradicional de la "difamación" de Crispo por parte de Fausta ( $\delta 1 \alpha \beta 0 \lambda \alpha \tilde{\iota} \varsigma \tau \tilde{\eta} \varsigma$ $\left.\mu \eta \tau \rho v i \tilde{\alpha} \varsigma^{35}\right)$. El Epitome de Caesaribus, una de las fuentes paganas más antiguas de la leyenda negra, menciona sin embargo que Constantino mató a Crispo "por sugestión de la emperatriz". La frase podría sugerir quizás la "difamación" de

${ }^{35}$ Tal como aparece, por ejemplo, en Philostorgius. Historia ecclesiastica (fragmenta ap. Photium)

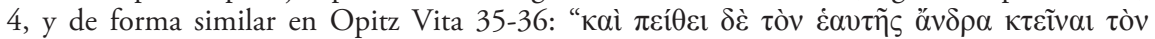

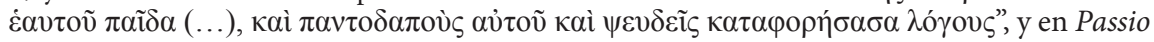

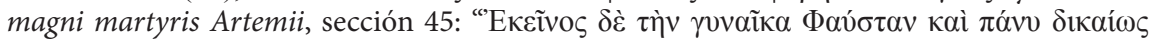

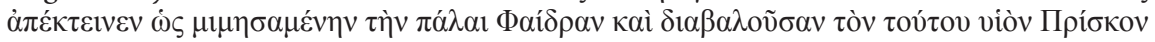

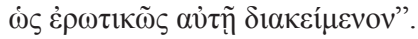


Fausta propia del mito de Fedra, aunque la alusión es demasiado ambigua para poder afirmarlo: en primer lugar, la idea de "sugestión" no se acerca exactamente al motivo de la difamación; en segundo lugar, incluso una referencia explícita a la "difamación" puede tener un significado diferente al que se le da en el marco del mito de Fedra: la emperatriz podría haber difamado al césar diciendo que éste pretendía, por caso, apropiarse del trono imperial, como de hecho se ha sugerido ${ }^{36}$.

Aunque no resulte posible establecer con certeza si la utilización del mito de Fedra es de origen arriano, es interesante notar en todo caso el detalle con que Philostorgius parece haberse detenido a exponer las circunstancias de las muertes del césar y la emperatriz. El hecho es significativo porque, siguiendo de cerca la estructura del mito, las tres versiones dejan en claro la inocencia de Crispo -el joven habría rechazado el amor de Fausta, como correspondía a un hijo virtuoso, pero había sido castigado a pesar de todo, lo cual reforzaba aún más el injusto comportamiento de Constantino. Más aún: si bien la Artemii Passio se contentaba con explicar la muerte de Fausta aduciendo (como las versiones paganas) que el emperador la habría castigado de esa forma luego de haber descubierto sus mentiras respecto a Crispo, la Opitz Vita y Photios preservaron una versión de los hechos aún más insidiosa: de acuerdo con ella, la emperatriz, luego de haber calumniado a Crispo y haber logrado su muerte, se habría enamorado de uno de los hombres del emperador, un cursor, con quien habría mantenido una relación que habría sido descubierta por Constantino y castigada con la muerte ${ }^{37}$. En apariencia, los arrianos habrían definido así una imagen del emperador que, en algunos aspectos, era aún más crítica e incluso más degradante que las versiones paganas.

Más allá de eso, es interesante notar que si bien Philostorgius ofrecía una versión de las muertes de Crispo y Fausta que hacía explícita la culpabilidad del emperador ${ }^{38}$, no por eso vinculaba la conversión de Constantino al arrepentimiento por sus crímenes, como hacían las versiones paganas. De hecho, Philostorgius retomaba el motivo de la visión de la cruz durante la guerra contra Majencio y lo presentaba, a la manera de la leyenda cristiana, como el momento de la conversión del emperador ${ }^{39}$. En apariencia, la degradación de la figura

36 N. J. E. Austin, “Constantine and Crispus”, pp. 133-8.

${ }^{37}$ Philostorgius. Kirchengeschichte, II.2; Opitz Vita, 36.

38 Esa versión de los crímenes de Constantino se ve, desde ya, deformada en la Artemii Passio, que reordena la información tomada de Philostorgius para hacer una defensa del emperador frente a una difamación pagana identificada con Juliano el Apóstata. Entre líneas, sin embargo, debe leerse la crítica original del arriano Philostorgius a Constantino.

${ }^{39}$ Cf., por ejemplo, Opitz Vita, 20. 
imperial empezaba para la tradición arriana en el concilio de Nicea I, donde el emperador se habría alejado de la verdadera fe; luego, ya volcado a la herejía, habría cometido otros crímenes, como los asesinatos de su hijo y su esposa.

La leyenda arriana no agregaba así temas diferentes, o potencialmente más peligrosos, a la leyenda pagana de Constantino. Pero había algunos aspectos de la obra de Philostorgius que, más allá de la leyenda, podían resultar especialmente perjudiciales para la figura del emperador santo. El más importante de ellos era la vinculación entre Constantino y Eusebio de Nicomedia. Photios señalaba en su Biblioteca la alta estima en que Philostorgius tenía al obispo arriano de Nicomedia - “a quien llama 'el Grande' por sus portentos y su vida”-40, y, a pesar de que la obra no parece haber enfatizado el bautismo de Constantino por Eusebio al final de su vida, sí menciona la confianza del emperador en el obispo de Nicomedia, a quien le habría confiado el testamento que establecía la venganza a tomar contra sus hermanos (sus supuestos asesinos) y la repartición del Imperio entre sus hijos ${ }^{41}$. Y esa referencia tenía la capacidad de preocupar especialmente a los autores cristianos en la medida en que, lejos de la leyenda arriana, evocaba un hecho histórico de la vida del emperador, un hecho que había sido además incorporado a la propia leyenda cristiana a través de la Vita Constantini de Eusebio de Cesárea. Para los autores cristianos del siglo VII en adelante, el interés fundamental no sería ya desvincular al emperador de las muertes dudosas de Crispo y Fausta, sino desvincularlo de su heterodoxa relación al arrianismo.

\subsection{La (nueva) leyenda cristiana}

Las referencias al bautismo en Nicomedia poco antes dela muerte del emperador no tenían, en la Vita Constantini de Eusebio, ninguna connotación particular: el bautismo tardío era todavía una práctica corriente en la época, y el hecho de que el sacramento hubiese sido impartido por un obispo arriano no podía tener para Eusebio, arriano moderado él mismo, ningún aspecto objetable ${ }^{42}$. Los sucesores inmediatos de Eusebio -Sócrates, Sozómeno y Teodoreto- seguirían la misma versión de los hechos a pesar de su adscripción nicena, y dos de ellos recordarían además que Constantino había confiado su testamento al obispo de Nicomedia, con la orden de no entregarlo a nadie más que a su hijo Constancio ${ }^{43}$; aún en

40 R. Henry, Photius, Bibliothèque, Les Belles Lettres, Paris, 1959-1977, codex 40 (8b).

${ }^{41}$ Philostorgius. Kirchengeschichte (fragmenta ap. Photium), II.16.

42 Über das Leben des Kaisers Konstantin, IV.61-63.

43 Sozomenus. Kirchengeschichte, II.34: "[Constantino] colocó su testamento en las manos del 
el siglo VI, como se ha visto antes, Evagrio Escolástico enfatizaría el bautismo tardío en Nicomedia para disociarlo de las muertes de Crispo y Fausta ${ }^{44}$.

Ya para el siglo VIII, sin embargo, el motivo del bautismo sería objeto de preocupación para los autores cristianos. La posibilidad de que Constantino hubiese sido bautizado por un obispo arriano se veía agravada por las implicancias derivadas del bautismo tardío: la Iglesia habría sido regida por un emperador no bautizado, y el propio concilio de Nicea I -convocado, presidido y guiado Constantino- perdía así parte de su legitimidad, como lo sugería la crónica de Teophanes:

“(...) los del este dicen que [Constantino] fue bautizado en Nicomedia hacia el final de su vida por el arriano Eusebio de Nicomedia, y que allí murió. Y esto debido a que había postergado el bautismo esperando poder ser bautizado en el río Jordán. A mí me parece más verosímil que haya sido bautizado en Roma por Silvestre y que las disposiciones dadas a Milcíades en su nombre sean falsificaciones de los arrianos, que se esforzaban así por preservar su doctrina y pretendían difamar al virtuoso emperador, presentándolo como una persona no bautizada y por ende indigna de confianza y mentirosa. Pues, si no hubiera estado bautizado, ¿cómo podría haber participado en el concilio de Nicea, cómo podría haberse unido a las plegarias de los santos padres? (cỉ yà $\rho$

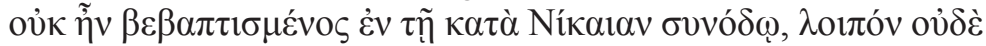

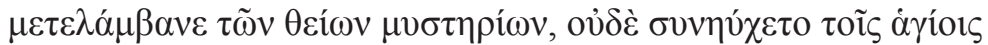
$\pi \alpha \tau \rho \alpha ́ \sigma ı v)$. Semejante cosa es absurda de decir y de pensar" ${ }^{45}$.

La Guidi Vita Constantini del siglo IX, la crónica de Cedrenus del siglo XI y la Historia Ecclesiastica de Kallistos Xanthopoulos del siglo XIV repetían el mismo planteo de Teophanes, con variaciones menores ${ }^{46}$. Una vez más, los autores cristianos se veían en la necesidad de reformular aspectos (esta vez

presbítero que permanentemente exaltaba a Arrio, y que le había sido recomendado como un hombre virtuoso por su hermana Constancia al momento de su muerte; le encargó, luego de haberle hecho prestar juramento, que entregase el testamento a su hijo Constancio"; Socrate de Constantinople, Histoire ecclésiastique, I.39: “(...) y confiando la custodia de su testamento al presbítero por intermedio del cual Arrio había sido llamado de regreso (...) le encargó que no lo entregase a nadie más que a su hijo Constancio".

${ }^{44}$ The ecclesiastical history, p.141.

${ }^{45}$ Theophanis Chronographia, pp.17-18.

${ }^{46}$ Guidi Vita, sección 18; Georgius Cedrenus opera, vol. I, p. 476; Nicephorus Callistus Xanthopoulos, Historia ecclesiastica, VII.35. 
históricos) de la vida de Constantino, para adecuarlos a la tradición ortodoxa del emperador santo. Y fue justamente en función de esa doble necesidad de distanciar a Constantino de los elementos paganos y arrianos asociados a su figura que la leyenda papal de la Vita Silvestri fue fácilmente incorporada en Bizancio: frente a una tradición oriental compleja e imperfectamente definida, la cancillería romana ofrecía -en el motivo de la revelación de Pedro y Pablo y del bautismo por el papa Silvestre- la posibilidad de preservar la esencia de la santidad imperial ${ }^{47}$. A partir de su primera difusión en el siglo VI, los motivos de la conversión y el bautismo definidos por la Vita Silvestri pasarían a ser los tradicionales en la leyenda ortodoxa de San Constantino.

\section{Conclusión}

La construcción de la santidad de Constantino I responde así a un proceso complejo en el cual resulta necesario detenerse para observar en qué medida la figura del primer emperador santo constituye un ejemplo propio de santidad definida en términos políticos. Más allá de la disputa pagano-cristiana en torno a Constantino -cuyo carácter político resulta evidente-, es interesante considerar la transición en virtud de la cual la santidad imperial pasó a constituir un motivo de disputa al interior del propio cristianismo. El caso de Constantino I frente al arrianismo no haría más que ofrecer, en ese sentido, un primer ejemplo dentro de una larga lista de emperadores cuya santidad -como la de Teodosio I, Marciano, León I, Justino I, Justiniano I y II, Constantino IV- iba a estar vinculada a la definición de la Ortodoxia cristiana.

La importancia del emperador bizantino en la definición de la Ortodoxia -convocatoria a concilios, participación y frecuente liderazgo en reuniones conciliares, reafirmación de una línea teológica- implicaría necesariamente que la figura imperial iba verse involucrada de forma permanente en controversias de tipo teológico-político. En numerosas ocasiones, la figura imperial sería así árbitro de disputas intra-eclesiásticas (muchas veces geo-eclesiológicas) que opondrían a distintos sectores de la Iglesia en torno a un problema cuyo carácter era superficialmente teológico pero en esencia político: en su calidad de árbitro, el emperador favorecería a un sector de la Iglesia -como es el caso de Constantino I en Nicea- y sería con frecuencia santificado por la facción triunfante.

${ }^{47}$ Las implicancias eclesiológicas de la Vita Silvestri tuvieron escasa significación en Bizancio. Cf. Dagron G., Empereur et prêtre, pp.156-159. 
La santidad imperial se vería así generalmente definida en función de (y al servicio de) una línea teológica en particular, como lo ilustra el caso de la "ortodoxia nicena" de san Constantino. Es cierto que la santidad de Constantino es particularmente compleja -en parte por las derivas arrianas del propio emperador, en parte porque varios de los sucesores de Constantino fueron arrianos y porque el credo niceno no fue oficialmente ratificado hasta el 381-, pero, en todo caso, su figura ofrece un ejemplo ilustrativo del rol teológicopolítico que habría de jugar, desde los mismo orígenes del Imperio cristiano, la santidad de los emperadores.

\section{Referencias bibliográficas}

Adler, A. (ed.) (1928-1935), Suidae lexicon, Leipzig, Teubner.

Amidon, P. (trad.) (2007), Philostorgius: Church History, Atlanta, Society of Biblical Literature, N. J. E. Austin (1980), "Constantine and Crispus", Acta Classica 23, pp. 133-8.

Barnes, T. (1981), Constantine and Eusebius, Cambridge Mass., Harvard University Press, p. 220;

Bekker, I. (ed.) (1836), Michaelis Glycae annales [Corpus scriptorum historiae Byzantinae], Weber, Bonn. (1838-1839) Georgius Cedrenus opera [Corpus scriptorum historiae Byzantinae], Weber, Bonn.

Bidez J. y Hansen, G. C. (eds.) (1960), Sozomenus. Kirchengeschichte [Die griechischen christlichen Schriftsteller 50], Akademie Verlag, Berlin.

Bidez J. y Partmentier, L., (1898), The ecclesiastical history of Evagrius with the scholia. London, Methuen.

Büttner-Wobst, T. (ed.) (1897), Ioannis Zonarae epitomae historiarum libri xviii, vol.3 [Corpus scriptorum historiae Byzantinae], Weber, Bonn.

Dagron, G. (1984), Constantinople Imaginaire. Etudes sur le recueil des Patria, Presse Universitaire de France, Paris. (1996) Empereur et prêtre. Étude sur le "césaropapisme" byzantin, Gallimard, Paris.

De Boor, C. (ed.) (1904), Georgii monachi chronicon, 2 vols., Teubner, Leipzig.

De Boor, C. (ed.) (1883), Theophanis Chronographia, vol. 1, Teubner, Leipzig. 
Drujvers, J. W. (1992), "Flavia Maxima Fausta: Some Remarks", Historia, 41, pp. 500-6.

Courcelle, P. y Loyen, A. (eds.) (1970), Sidoine Apollinaire, Lettres, Les Belles Lettres, Paris.

Guthrie, P. (1966), "The Execution of Crispus”, Phoenix, 20, pp. 325-31.

Hansen, G.C. (ed.) (1995), Theodoros Anagnostes. Kirchengeschichte 2nd edn. [Die griechischen christlichen Schriftsteller der ersten Jahrhunderte. Neue Folge 3], Akademie Verlag, Berlin.

Henry, R. (ed.) (1959-1977), Photius, Bibliothèque, Les Belles Lettres, Paris.

Kotter, P. B. (ed.) (1988), Passio magni martyris Artemii, en Die Schriften des Johannes von Damaskos, vol. 5 [Patristiche Texte und Studien 29], De Gruyter, Berlin-New York.

Lacombrade, Ch. (ed.) (1964), Simposion, en L'empereur Julien. Ouvres complètes, Les Belles Lettres, Paris.

Lieu, S. N. C. y Montserrat, D. (eds.) (1996), From Constantine to Julian: Pagan and Byzantine Views. A Source History, Londres - Nueva York, Routledge.

Maraval, P. y PÉrichon, P. (2004-2007), Socrate de Constantinople, Histoire ecclésiastique (Livres I-VII), Éditions du Cerf, Paris.

Migne, J.-P. (ed.) (1857-1866), Nicephorus Callistus Xanthopoulos, Historia ecclesiastica [Patrologiae cursus completus series Graeca], Paris.

Opitz, H.-G. (1934), “Die Vita Constantini des Codex Angelicus 22”, Byzantion 9, pp 535-93.

Parmentier, L. y Scheidweiler, F. (eds.) (1954), Theodoret. Kirchengeschichte [Die griechischen christlichen Schrifsteller 44], Akademie Verlag, Berlin.

Paschoud, F. (ed.) (1971-1989), Zosime. Histoire nouvelle, Les Belles Lettres, Paris.

Pichlmayr, F. (ed.) (1911), Sexti Aurelii Victoris Liber de Caesaribus. Praecedunt Origo gentis Romanae et Liber de viris illustribus urbis Romae; subsequitur Epitome de Caesaribus, Leipzig, Teubner.

Pohlsander, H. A. (1984), “Crispus: Brillant Career and Tragic End”, Historia 33, pp. 79-106.

Rougé, J., (1980), "Fausta, femme de Constantin: criminelle ou victime?", Cahiers d'Histoire, 25, pp. 3-17. 
Van Dam, R. (2011), Remembering Constantine at the Milvian Bridge, New York, Cambridge University Press.

Winkelmann, F. (ed.) (1975), Eusebius Werke, Band. 1.1: Über das Leben des Kaisers Konstantin [Die griechischen christlichen Schriftsteller], Akademie Verlag, Berlin. (1981) Philostorgius. Kirchengeschichte (fragmenta ap. Photium) [Die griechischen christlichen Schriftsteller], Akademie Verlag.

Woods, D. (abril 1998), "On the death of the Empress Fausta", Greece and Rome, 45, pp. 70-86. 